\title{
Quaderni
}

QUADERNI Communication, technologies, pouvoir

67| Automne 2008

Jeu vidéo et discours

\section{Violence, cinéma et jeux vidéo : de la récurrence d'un même discours}

Violence, cinema and videogames: from the recurrence of a same discourse

\section{Alexis Blanchet}

\section{(2) OpenEdition}

1 Journals

\section{Édition électronique}

URL : http://journals.openedition.org/quaderni/188

DOI : $10.4000 /$ quaderni. 188

ISSN : 2105-2956

\section{Éditeur}

Les éditions de la Maison des sciences de l'Homme

\section{Édition imprimée}

Date de publication : 5 octobre 2008

Pagination : 11-18

\section{Référence électronique}

Alexis Blanchet, «Violence, cinéma et jeux vidéo : de la récurrence d'un même discours 》, Quaderni [En ligne], 67 | Automne 2008, mis en ligne le 05 janvier 2012, consulté le 30 avril 2019. URL : http:// journals.openedition.org/quaderni/188; DOI : 10.4000/quaderni.188 


\section{$D$ ossier}

\section{violence, \\ cinéma et \\ jeux vidéo : \\ de la récurrence}

\section{d'un même discours}

\section{Alexis Blanchet}

ATER en Études cinématographiques Université Paris Ouest Nanterre chercheur associé à la BnF
En 1976, l'éditeur américain Exidy crée le jeu Death Race inspiré du film de série B Death Race 2000, de Paul Bartel ${ }^{1}$, produit par Roger Corman. Intitulé à l'origine Pedestrian, ce jeu vidéo invite le joueur à piloter à l'aide d'un volant et d'une pédale d'accélération une voiture sur une aire de jeu limitée au cadre de l'écran où des piétons apparaissent aléatoirement. Ceux-ci rapportent des points s'ils sont écrasés, se transformant dès lors en pierre tombale que le joueur doit ensuite éviter au risque de perdre la partie. Selon le score atteint, le joueur obtient le niveau de Chasseur de squelettes, de Briseur d'os ou mieux encore de Pilote expert. On relèvera que la situation de jeu est empruntée à une des idées chocs du scénario du film source soulignée par la phrase d'accroche ou tagline de l'affiche : «En l'an 2000, heurter les piétons sans s'arrêter n'est plus considéré comme un délit. C'est un sport national ! $»^{2}$ Dans cette course folle à travers les États-Unis, les piétons renversés rapportent en effet aux compétiteurs un certain nombre de points calculés selon un macabre barème basé sur l'âge des victimes.

Ainsi, dans une séquence particulièrement féroce du film, l'on peut voir une jeune fille se jeter sous les roues de la voiture de l'un des concurrents dénommé Frankenstein en raison de multiples opérations subies après plusieurs accidents en course. Vouant un véritable culte à ce pilote vedette et portée par une ferveur sûrement excessive, elle se sacrifie ainsi dans le but de rapporter quelques précieux points à son héros sportif.

Si le film peut paraître ambigu - une dénonciation de la violence d'état par un appel à une violence visuelle et thématique qui peut choquer -, il n'en reste pas moins une fable plutôt amusante 
sur les dérives de la télévision et la crainte de nouveaux totalitarismes. Il rejoint en cela des films d'anticipation comme Rollerball de Norman Jewison sorti la même année et auquel il emprunte clairement sa thématique, Le Prix $d u$ danger, d'Yves Boisset réalisé en 1983 ou l'adaptation du roman de Stephen King, The Running Man, par Paul Michael Glaser en 1987 ; chacun de ces films invitant, avec plus ou moins de finesse et d'intelligence, à une réflexion sur le contrôle des foules par le spectacle télévisé d'une violence débridée.

Quelques ligues de vertu et associations familiales américaines attaquèrent violemment le jeu Death Race en raison de la brutalité et de l'immoralité de la situation ludique proposée au joueur. Ainsi, le National Safety Council's, une organisation fondée en 1913 très impliquée dans la prévention routière, jugea à sa sortie le jeu « malade, perfide et morbide $»^{4}$.

Afin de préserver une jeunesse s'adonnant régulièrement à ce type de divertissement, mais jugée facilement influençable et trop vulnérable à la puissance de ces nouvelles images, les contempteurs du jeu demandèrent purement et simplement son interdiction immédiate. Si la commercialisation des bornes d'arcade du jeu ne fut pas totalement arrêtée, Exidy ralentit cependant leur diffusion le temps que se taisent ces protestations. La polémique créée à propos du jeu lui valut quelques temps plus tard une citation dans le magazine télévisé d'investigation 60 minutes de la chaîne CBS consacré alors aux dangers psychologiques de la pratique du jeu vidéo. Cette soudaine médiatisation, accompagnée d'articles dans plusieurs titres de presse dont le National
Enquirer, aurait finalement permis à Exidy de relancer la production de bornes d'arcade Death Race passant des 500 exemplaires initialement commercialisé à près de $10000^{5}$ confirmant ainsi qu'une campagne de presse, même - et surtout? - mauvaise pouvait grandement aider au succès commercial de produits à destination d'un public adolescent.

À notre connaissance, le film source quant à lui n'a suscité aucune réaction à sa sortie : classé Restricted par la MPAA (Motion Picture Association of America ), l'instance de classification des films de cinéma produits et diffusés aux États-Unis, le système d'autorégulation mise en place par Hollywood a de fait limité son accès aux salles traditionnelles. Aux États-Unis, les films classés R ou NC-17 sont quasiment condamnés commercialement car les réseaux de salle soumis aux classifications MPAA n'assument que très rarement le risque de les programmer.Cantonné aux drive-in (sûrement aidé en cela par sa thématique automobile) et aux salles de projection indépendantes spécialisée, dans le cinéma d'exploitation, Death Race 2000 est donc vraisemblablement passé totalement inaperçu auprès des mêmes groupes de pression.

La déclinaison en jeu vidéo de Death Race 2000 sera donc, seule, objet de polémique. Dans l'histoire naissante du jeu vidéo, cette levée de boucliers à l'encontre d'un logiciel est le premier exemple identifié d'une controverse sur la violence supposée et les risques liés aux jeux vidéo. Or, pour qui a déjà joué à Death Race ou vu un écran de jeu du logiciel incriminé - quelques images du jeu d'Exidy apparaissent non sans une certaine ironie dans deux plans du film Jaws II 
(en français Les dents de la mer II), de Jeannot Swarc -, la violence des images du jeu apparaîtra somme toute relative : les capacités graphiques du matériel rudimentaire de l'époque permettant, en noir et blanc, une figuration toute symbolique de corps passant sous les roues des véhicules. Quels aspects du jeu ont pu déclencher l'ire de ses détracteurs? Est-ce bien une dénonciation de la violence de l'œuvre qui est au cœur de ces attaques ou un autre enjeu, moins apparent, peutil expliquer cette montée au créneau d'une partie des tenants de la moralité ?

Le propos de cet article est donc d'interroger la récurrence de discours sur la violence du cinéma comme du jeu vidéo et leurs effets supposés sur le public, en s'interrogeant sur les contextes dans lesquels ces discours apparaissent et en saisissant les raisons pour lesquelles les mêmes arguments sont employés à propos de différents médias à plusieurs dizaines d'années d'intervalle. Devant l'impossibilité à embrasser ici dans leur globalité les histoires de ces deux médias, celle du cinéma aujourd'hui plus que centenaire et celle du jeu vidéo longue de bientôt quarante ans, nous nous attarderons d'une part sur l'accueil du film de gangster aux Etats-Unis dans les années 1930 et d'autre part les propos tenus à l'encontre de quelques jeux vidéo des années 1980 et 1990 .

Ces discours présentent ainsi de nombreuses similitudes argumentaires quant à la question de la violence accompagnant l'émergence de la pratique grand public d'un nouveau média. Cette réflexion historique vise surtout à mettre en lumière un certain parallélisme observé dans les discours concernant les prétendus dangers relatifs au rapport à la fiction.
Dans son ouvrage consacré au Pre-Code Hollywood, Thomas Doherty soutient que le cinéma hollywoodien classique, période que les historiens du cinéma font débuter au cours des années 1930 et aboutir à la fin des années 1950, a eu un impact culturel décisif et tout à fait inestimable sur la manière de concevoir et de recevoir les contenus audiovisuels contemporains. Ainsi l'industrie du divertissement américain et le «kaléidoscope mondial que composent films, programmes de télévision, jeux vidéo, images de synthèse et autres $C D-R O M »$ ne fonctionnent que sur l'héritage fécond et pourtant en partie censuré de la production hollywoodienne classique : thèmes, matière et contextes fictionnels, structures des récits, mais aussi un très lourd legs moral ${ }^{6}$.

Or, l'Hollywood classique se construit lui-même sur un court intervalle de quatre ans qui sépare la mise en œuvre aux débuts du parlant d'un code de « bonne conduite » de l'industrie cinématographique en 1930 de sa réelle application en $1934^{7}$. Si les studios hollywoodiens doivent, depuis le début du $\mathrm{XX}^{\mathrm{e}}$ siècle et la mise en place du système des studios, faire face aux demandes récurrentes de la part de différents groupes de pression, d'interdiction de certains films et d'une manière plus générale d'une moralisation de la production dans son ensemble ${ }^{8}$, ces quatre années constituent un temps particulièrement marqué par les débats et les controverses sur les films proposés alors au public par Hollywood.

Ce Pre-Code Hollywood, comme le nomme Thomas Doherty, se caractérise autant en termes d'inventivité, de prise de liberté et de création cinématographique, que de polémiques à propos du contenu des films : dans une période marquée 
par les effets de la crise de 1929 et par le retour d'une rigueur tant morale qu'économique, les films hollywoodiens tentent de lutter contre la baisse de fréquentation des salles en convoquant des thématiques attrayantes, troublantes et souvent peu morales. Avec leurs connotations sexuelles explicites ou encore le recours aux motifs violents, ces œuvres surprennent encore aujourd'hui par leur hardiesse.

Alors que la prohibition a toujours cours et que le taux de chômage est au plus haut, un genre en particulier invite le public du cinéma dans un monde d'opulence, de luxe et d'excès : le film de gangster construit son succès sur l'ascension rapide et la chute vertigineuse («the rise and fall ») de jeunes gens ambitieux dans l'univers du crime organisé. Il met en scène des fusillades, des règlements de compte, des bastonnades, mais donne aussi à voir des fêtes fastueuses dans des lieux somptueux. À l'heure où le modèle capitaliste américain semble totalement à l'arrêt, les gangsters semblent être les derniers à pouvoir faire vivre le mythe du self made man dans l'American Dream.

Les genres cinématographiques « à problèmes » sont, comme le souligne Stephen Prince', l'horror et les crime movies. Cette seconde catégorie dont l'appellation même insiste sur le motif problématique, c'est-à-dire le crime donné à voir sous toutes ses formes, est au cœur de la polémique. Scarface, de Howard Hawks (1932) qui forme avec Little Caesar, de Mervyn LeRoy (1931) et The Public Enemy, de William A. Wellman (1931) les trois œuvres emblématiques du genre, fut ainsi durement attaqué à sa sortie. Comme le remarque avec ironie Thomas Doherty, la violence sans précédent à l'époque de Scarface «choqua et offensa éditorialistes, politiciens, et, semble-t-il, quiconque à portée de main d'un stylo ou d'une machine à écrire ». Selon Jack Alicoate, journaliste au Film Daily, un organe pourtant davantage consacré à l'économie de l'industrie qu'à la critique, le film de Hawks distillait « un sentiment aigu de nausée », l'action y étant «d'une intensité si aliénante qu'elle ne laisse aucun répit au spectateur », son unique suspense résidant dans le fait de savoir «quelle allait être la brutalité nouvelle employée pour le prochain massacre. » Et Alicoate de poursuivre : «Il y a certaines choses qui ne devraient pas apparaître sur les écrans de cinéma. (...) La matière même du sujet de Scarface en est l'une d'elles. Le projeter sans discernement sur les écrans d'Amérique fera du tort à l'industrie $d u$ cinéma toute entière ainsi qu'aux personnes qui collaborent avec elle. (...) Ce film n'aurait jamais du être tourné. $»^{10}$

Scarface fait aussi partie des contre-exemples de moralité analysés par Martin Quigley parmi plusieurs films distribués de 1932 à 1934, dans un ouvrage publié en 1937, Decency in Motion Pictures. Quigley, éditeur du Motion Picture Herald et rédacteur avec le père jésuite Daniel Lord du code de production, y relève qu'au contraire du représentant de la loi, le criminel y est montré comme « riche, courageux et rusé ${ }^{11}$ et se désespère d'y voir présentées les méthodes qu'utilisent les criminels.

Comme le note Jean-Loup Bourget, les « libertés » prises par certains films du début des années 1930 eurent pour conséquence de stimuler les ligues de vertu, «c'est-à-dire avant tout la Legion 
of Decency, puissante organisation qu'encadrait la hiérarchie catholique elle-même dominée par des Irlandais conservateurs. Les catholiques sont aux États-Unis nettement minoritaires, mais ils constituent un bloc assez soudé, bien organisé ; les mots d'ordre de la hiérarchie sont diffusés avec rapidité. Aussi donnèrent-ils le signal; les ligues de vertu protestantes applaudirent et emboîtèrent le pas. La menace était grave : des millions de "légionnaires 》 ou de "croisés " faisaient vou de boycotter les films taxés d'immoralité. $\gg{ }^{12}$

Chaque fait de délinquance juvénile devient alors, comme le rappelle Thomas Doherty, l'occasion pour les églises, les groupes civiques et les politiciens d'intensifier leurs critiques à l'encontre de l'industrie et du film de gangsters dont l'influence, irrémédiablement néfaste, est considérée comme facteur déclencheur d'acte de violence : «Après qu'un adolescent meurtrier à East Orange, New Jersey, se soit dit influencé par Hollywood, le maire en appela à Hays lui-même pour interdire le genre gangster. À Worcester, Massachusetts, le chef de la police locale prit les choses en main en adressant une directive à l'intention des exploitants pour les informer que " dorénavant les films de gangsters ne seraient plus approuvés par [son autorité] lors des projections dans les salles de cinéma locales. $\rangle^{13}$

Enfin, les groupes de pression n'hésitent pas à appuyer leurs réserves et leurs critiques à l'encontre du cinéma sur des études scientifiques menées en sciences humaines. Comme le rappelle Jean-Loup Bourget, "l'offensive [menée contre l'industrie] était alimentée par les travaux de certains sociologues et psychologues qui rédigèrent une série de brochure, d'apparence scientifique, mais de visée polémique, pour démontrer les effets nocifs du cinéma, particulièrement sur les enfants et les adolescents. On dénonçait dans le cinéma l'école de la violence en même temps que du vice. ». Parmi les exemples les plus significatifs de cette instrumentalisation de la recherche scientifique au profit de thèses hostiles au cinéma, on retrouve le cas des Payne Fund Studies, une enquête sociologique d'envergure conduite par le Motion Picture Research Council pour comprendre l'influence des films sur les enfants. Des neuf volumes de ce travail colossal et rigoureux mené de 1928 à 1933, Henry James Forman tire en 1934 un livre par trop simpliste et bien moins nuancé, au titre alarmiste «Our movie made children » («Nos enfants façonnés par les films »), destiné à devenir un best seller ${ }^{14}$. L'ouvrage se donne clairement pour objectif de constituer le film de cinéma en problème social et tente d'influencer les leaders d'opinion par une campagne de presse savamment orchestrée ${ }^{15}$. Rapportées par les journaux pour le grand public, les conclusions de Forman déjà fortement orientées subissent encore de nouvelles simplifications ; le mythe du média prescripteur et déclencheur de comportements violents est désormais bien ancré dans l'esprit du public américain et a vraisemblablement de beaux jours devant lui.

Car on le voit, ces discours sur la violence de films de gangsters et les réactions médiatiques et politiques que ceux-ci provoquent nous rappellent fortement ce que l'on a pu observer dans les années 1980 et 1990 à propos de jeux vidéo comme Mortal Kombat d'Acclaim, Doom d'ID Software ou Grand Theft Auto de Rockstar : dénonciation de la représentation et de la valorisation des actes 
de violence dans les œuvres incriminées, peur du risque d'imitation chez les jeunes, mise en cause des systèmes d'autorégulation mis en place par le secteur industriel, désir d'une moralisation des industries du divertissement et enfin appel à une autorité scientifique pour valider le discours catastrophiste.

Ce que révèle l'émergence de discours sur l'utilisation de la violence par un média, c'est avant tout le statut que ses contempteurs lui reconnaissent, à la fois l'aveu d'une influence médiatique indiscutable sur la société en tant que média de masse car en effet, le cinéma comme le jeu vidéo « contrairement à la musique et à la littérature, s'adresse aux masses », mais aussi la reconnaissance de ses capacités particulières de conviction, "de la différence d'impact entre le livre, qui décrit, et le film qui montre, ou encore du phénomène d'identification avec le protagoniste, aggravé par le fait que ce dernier est interprété par une star. $\rangle^{16}$

Ce qui vaut finalement tant de reproches au film de gangster des années 1930 et aux jeux vidéo à caractère violent, c'est avant tout leur appartenance au domaine de la fiction. En effet, cette dernière ressortit, pour Jean-Marie Schaeffer à cette activité de feintise qui se donne pour telle. Or, même si l'on sait pertinemment que les évènements vus sur un écran de cinéma ou joués sur l'écran d'une borne d'arcade sont les inventions de leur auteur, dont tout l'art est de nous les rendre convaincants et crédibles, la fiction est toujours suspectée d'avoir des effets sur la vie réelle. Comme le relève Schaeffer, deux périls pèsent depuis toujours sur la fiction dans la manière dont elle est considérée par la culture occidentale : «celui de l'immersion (donc de la perméabilité des frontières entre fiction et réalité) et celui de l'effet d'entraînement (donc de la modélisation de la réalité par la fiction). $»^{17}$

Alors que le débat fait rage sur le film de gangsters, les actualités cinématographiques donnent à voir à la même époque les dépouilles des gangsters abattus et des victimes de leurs forfaits : le corps de John Dillinger est ainsi filmé en juin 1934 à la morgue du Cook County Hospital et présenté au public quasi-immédiatement dans les salles; quelque temps plus tard, au mois de novembre, les dépouilles de Baby Face Nelson et de trois jeunes victimes retrouvées dans les Blue Ridge Mountains subissent le même traitement. Étonnamment, les groupes de pression réagissent moins fort à la violence crue de ces images, que même les exploitants hésitent à projeter en première partie de programme, redoutant de marquer l'esprit du spectateur d'une trace indélébile et, par conséquent, de gâcher le plaisir de la fiction qui suit. Les débats que provoque la diffusion de ces images tournent essentiellement sur la moralité de l'exploitation à des fins lucratives du spectacle de la mort par les majors. Le Motion Picture Herald s'interroge: «Les images de bandits tués et d'enfants assassinés rapportent-elles tant $d$ 'argent au box-office qu'elles puissent se passer des spectateurs les plus sensibles?»

Média apprécié des jeunes faisant largement appel aux mécaniques d'immersion dans son rapport à la fiction, le jeu vidéo présente donc, bien davantage que le cinéma, toutes les aptitudes pour être, en tant que média émergent, la cible d'attaques sur le thème de la violence. Si le jeu Death Race que nous évoquions en introduction 
de cette réflexion est tant décrié à sa sortie, c'est ainsi moins pour sa représentation graphique de la violence - dont nous avons vu qu'elle était largement émoussée par les capacités graphiques rudimentaires du dispositif -, que par la possibilité de piloter son avatar à l'écran au moyen d'un vrai volant et d'une vraie pédale d'accélération : la perméabilité entre la fiction vidéoludique et la réalité qu'autorise le dispositif n'est-il pas une invitation trop franche à reproduire l'impensable sur la route?

Remerciements à Francis Bordat
$\mathrm{N} \cdot \mathrm{O} \cdot \mathrm{T} \cdot \mathrm{E} \cdot \mathrm{S}$

1. Le film a été exploité en France sous deux titres : sa traduction littérale La course à la mort de l'an 2000, et aussi Les seigneurs de la route.

2. Citation en anglais tirée de l'affiche originale : «In The Year 2000 Hit And Run Driving Is No Longer A Felony. It's The National Sport! »

3. Le NSC se donnait alors et se donne d'ailleurs toujours pour but de «faire du monde un lieu plus $s \hat{u} r »$. Voir le site Internet http://www.nsc.org

4. « Sick, sick, sick », «Insidious » et « Morbid». Cité par Len Buckwalter dans son ouvrage Videogames: A Complete Guide, Tempo Books, New York, 1977.

5. D'après l'article en ligne souvent cité sur les controverses du jeu vidéo de Lauren Gonzalez, When Two Tribes Go to War: A History of Video Game Controversy, 2004, consultable en ligne à l'adresse : http://www.gamespot.com/features/6090892/index. html

6. Thomas Doherty, Pre-code Hollywood. Sex, immorality, and insurrection in American cinema 19301934, Columbia University Press, 1999. pp. 1-2

7. Le Production Code souvent appelé Code Hays du nom du président du Motion Picture Producers and Distributors of America (MPPDA), William H. Hays, est un texte dressant une liste de recommandations, mais aussi d'interdits acceptés par Hollywood sous la pression des défenseurs de la morale pour encadrer la production de films.

8. Jean-Loup Bourget, Hollywood, années 30. Du krach à Pearl Harbor, éditions des 5 continents/Hatier, Coll. Bibliothèque du cinéma, Paris, 1986. p. 29

9. Stephen Prince, Classical Film Violence. Designing and Regulating Brutality in Hollywood Cinema, 19301968, Rutgers University Press, New Brunswick, 
New Jersey, 2003.

10. Cité par Thomas Doherty, Ibid, p. 149

11. Cité par Jean-Loup Bourget, Ibid, p. 34

12. Idem, p. 34

13. Cité par Thomas Doherty, Ibid, p. 150

14. Richard Barrios, Screened OutPlaying Gay in Hollywood from Edison to Stonewall, Routledge, 2002. p. 130

15. Garth Jowett et al., Children and the Movies Media Influence and the Payne Fund Controversy, Cambridge University Press, 1996. p. 94

16. Jean-Loup Bourget, Ibid. p. 30

17. Jean-Marie Schaeffer, Pourquoi la fiction?, Paris, Le Seuil, 1999. p. 39
$R \cdot E ́ \cdot S \cdot U \cdot M \cdot E ́$

La récurrence de discours sur la violence du cinéma comme du jeu vidéo et leurs effets supposés sur le public, nous invite à nous interroger sur les contextes dans lesquels ces discours apparaissent et les raisons pour lesquelles les mêmes arguments sont employés à propos de différents médias à plusieurs dizaines d'années d'intervalle. L'accueil du film de gangster aux États-Unis dans les années 1930 et les propos tenus à l'encontre de quelques jeux vidéo des années 1980 et 1990 présentent ainsi de nombreuses similitudes argumentaires quant à la question de la violence accompagnant l'émergence de la pratique grand public d'un nouveau média. Cette réflexion historique vise surtout à mettre en lumière un certain parallélisme observé dans les discours concernant les prétendus dangers relatifs au rapport à la fiction.

Violence, cinema and videogames: from the recurrence of a same discourse

Speeches recurrence on violence as much on cinema as on videogames and their supposed effects on the public, invites us to analyse contexts in which they appear and to address the reasons why the same arguments are used about various media several decades apart. The gangster film reception in the 1930s' United States and the remarks made against the few games of the 1980s and 1990s have so many similar arguments on the issue of violence accompanying emergence of general public practice of a new medium. This primarily historical thinking is to highlight a certain parallelism observed in speeches concerning the alleged dangers of fiction. 